\title{
Poblaciones indígenas amazónicas en la pandemia de COVID-19
}

\author{
Amazonian indigenous populations in the COVID-19 pandemic
}

\author{
Sebastián Iglesias-Osores 1,a, Lizbeth Córdova-Rojas ${ }^{2, a}$
}

\section{INTRODUCCIÓN}

A nivel mundial, en todos los países y poblaciones, los pueblos indígenas enfrentan una mayor carga de enfermedad que los pueblos no indígenas, incluidas las enfermedades cardiovasculares y el $\mathrm{VIH}{ }^{(1)}$. La selva amazónica de Perú representa el $60 \%$ del territorio nacional y es el hogar de alrededor de 264 000 indígenas ${ }^{(2)}$.

Los estados indígenas amazónicos, podrían ser inusualmente vulnerables al COVID-19 debido a factores médicos, sociales y ambientales, como una alta prevalencia de tuberculosis y malaria, falta de agua potable y desnutrición ${ }^{(3)}$. Las enfermedades como dengue y zika son comunes en estos territorios ${ }^{(4)}$. Se encontró una alta prevalencia de diabetes, enfermedad renal crónica e hipertensión en una comunidad indígena ${ }^{(5)}$. Esto aunado a que se puede encontrar elevadas prevalencias de $\mathrm{VIH}$ y sífilis en algunas comunidades indígenas amazónicas agrava la pandemia por COVID-19 .

Se prevé que el impacto de la pandemia de COVID-19 será duradero con impactos intergeneracionales tanto para los pueblos indígenas como para los no indígenas ${ }^{(7)}$. Las fuerzas estructurales limitan el acceso a la atención médica y el racismo sistémico y la discriminación hacia los pueblos indígenas, pueden dificultar el desarrollo de relaciones de confianza con proveedores no indígenas ${ }^{(1)}$. La región amazónica tiene pocos hospitales con unidades de cuidados intensivos y un número limitado de ventiladores ${ }^{(3)}$.

Esta situación aumenta el riesgo de futuras emergencias por enfermedades infecciosas y reduce la posibilidad de obtener farmacopeas ancestrales, base de la práctica médica de los pueblos indígenas y potencial fuente de tratamiento para el resurgimiento de enfermedades infecciosas en la población futura. Su salud se ve afectada por factores de estrés epigenético, opresión y violencia generacional, incluido un número desproporcionado de mujeres indígenas desaparecidas y asesinadas, menor nivel educativo y pobreza persistente ${ }^{(1)}$. La deforestación y la fragmentación de los bosques son impulsadas por el aumento de explotación ilegal de la madera, las economías extractivas y la agricultura a gran escala (especialmente el aceite de palma africana y la hoja de coca) ${ }^{(8)}$.

La propagación mundial de COVID-19, ha generado respuestas médicas y de salud pública agresivas, que incluyen pruebas, detección, rastreo de contactos, distanciamiento social, restricciones de viaje y órdenes de quedarse en casa cuando se está enfermo o expuesto ${ }^{(9)}$. Esto se ha aplicado a muy pequeña o nula escala en los pueblos indígenas; en esta región existen alrededor de 42 millones de personas ${ }^{(10)}$. Las poblaciones indígenas prefieren muchas veces tratarse dentro de sus comunidades y por lo

\footnotetext{
${ }^{1}$ Facultad de Ciencias Biológicas, Universidad Nacional Pedro Ruiz Gallo, Lambayeque, Perú.

2 Universidad Nacional de Jaén, Jaén, Perú.

a Biólogo

Correspondencia: Sebastián Iglesias Osores
}

Correo: sebasiglo@gmail.com

Recibido: 27.06.2021 Aceptado: 01.09.2021 
tanto esta información se pierde y no es recabada por fuentes oficiales del estado, así como la falta de diagnóstico médico no se puede saber con certeza el número real de casos. Para rastrear las fuentes de infección y proteger a estas personas vulnerables, necesitamos que los datos sobre COVID-19 estén desglosados por etnia ${ }^{(11)}$. Los datos sobre la tasa de infección en los pueblos indígenas aún no están disponibles (incluso cuando se dispone de informes y pruebas) ${ }^{(12)}$.

\section{MÉTODOS}

Se obtuvo el número de casos confirmados de las comunidades indígenas amazónicas de sala de población indígena con COVID-19 elaborado por el CDC Perú - Minsa (https://www.dge.gob.pe/portalnuevo/informacion-publica/sala-de-poblacion-indigena-con-covid-19/). Ver tabla 1. Para el inicio de síntomas según la ficha epidemiológica ver la figura 1. Se puede observar el número de casos confirmados según etnia en la figura 2. Los fallecidos según etnia se pueden observar en la figura 3.

Tabla 1. Número de casos confirmados COVID-19 pertenecientes a comunidades indígenas amazónicas.

\begin{tabular}{lcc}
\hline Departamento residencia & $\mathbf{N}^{\circ}$ & $\mathbf{\%}$ \\
\hline Loreto & 7604 & 34,8 \\
Amazonas & 6781 & 31,1 \\
Ayacucho & 2 & 0,0 \\
Ucayali & 2709 & 12,4 \\
Puno & 0 & 0,0 \\
San Martín & 1665 & 7,6 \\
Junín & 1243 & 5,7 \\
Cusco & 429 & 2,0 \\
Pasco & 614 & 2,8 \\
La libertad & 2 & 0,0 \\
Madre de dios & 348 & 1,6 \\
Huánuco & 209 & 1,0 \\
Cajamarca & 188 & 0,9 \\
Ancash & 1 & 0,0 \\
Lima & 17 & 0,1 \\
Apurímac & 0 & 0,0 \\
Tacna & 1 & 0,0 \\
Huancavelica & 0 & 0,0 \\
Lambayeque & 13 & 0,1 \\
Arequipa & 0 & 0,0 \\
Callao & 1 & 0,0 \\
Moquegua & 1 & 0,0 \\
Ica & 21830 & 0,0 \\
Piura & 0,0 \\
Tumbes & 0,0 \\
Total & 0 \\
\hline
\end{tabular}




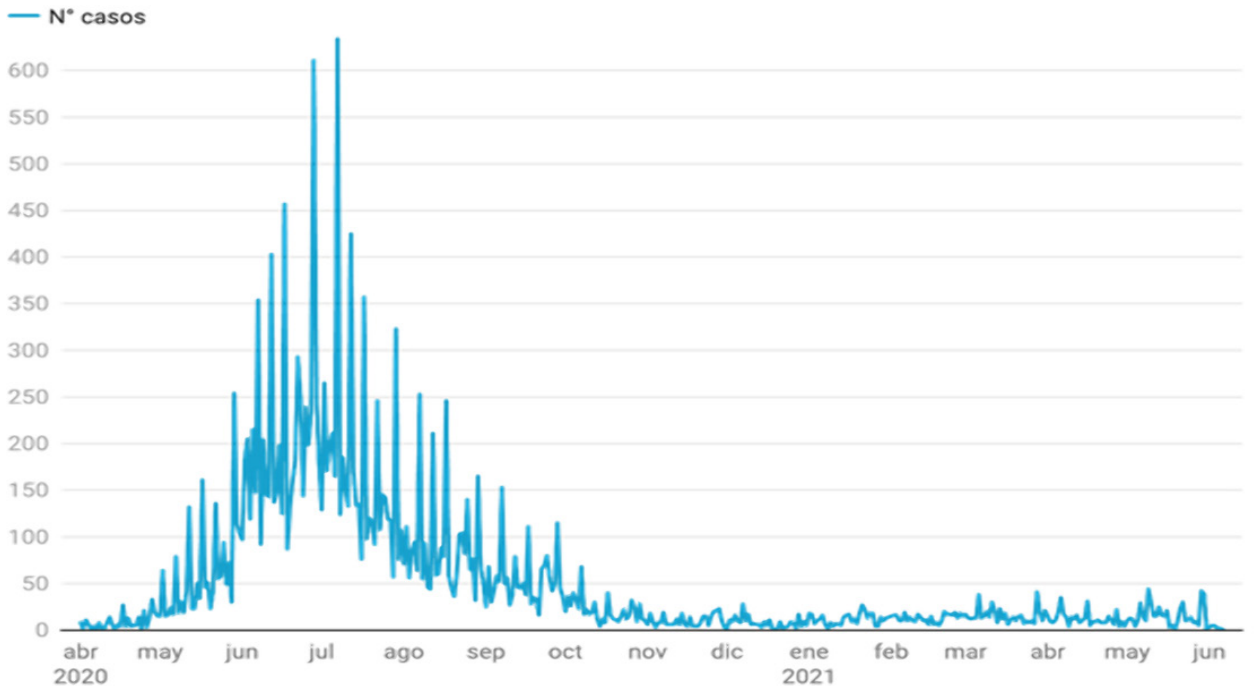

Figura 1. Fecha de inicio de síntomas según ficha epidemiológica de pacientes COVID-19 confirmados.

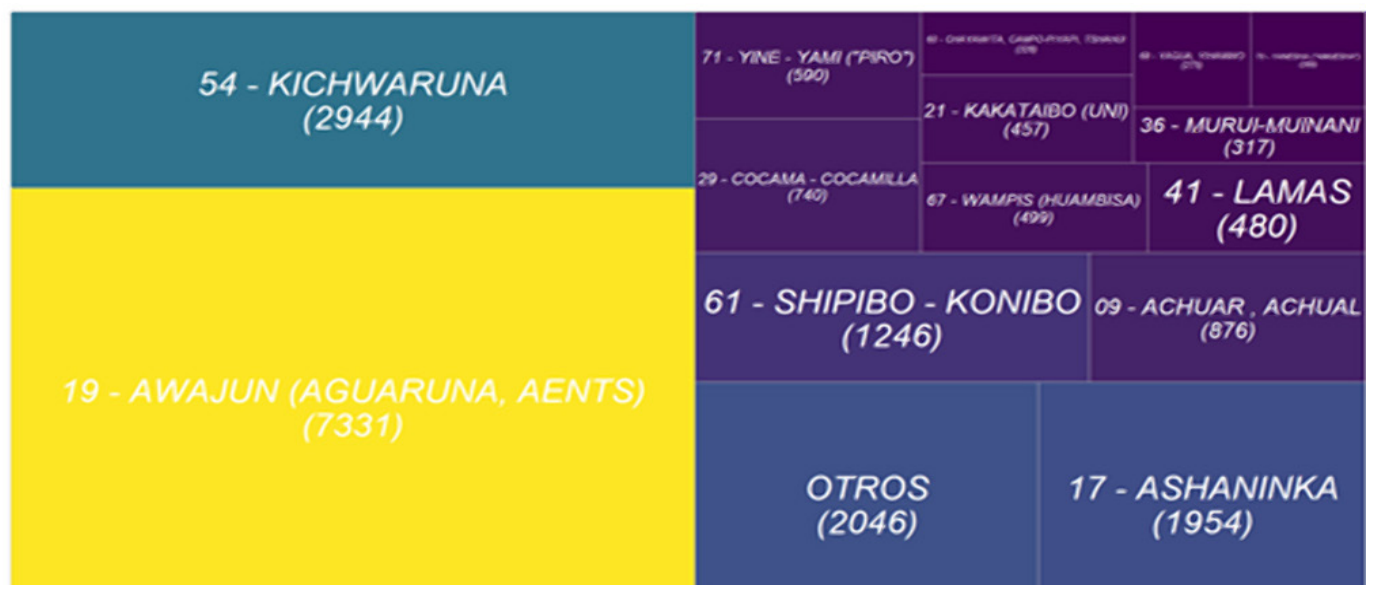

Figura 2. Número de casos confirmados COVID-19 de comunidades indígenas amazónicas según etnia.



Figura 3. Número de fallecidos confirmados COVID-19 de comunidades indígenas amazónicas según etnia. 
Según los datos obtenidos por el Centro Nacional de Epidemiología, Prevención y Control de Enfermedades (CDC-Perú), la región con más casos confirmados de COVID-19 fue Loreto (figura 4) seguida de Amazonas. La etnia Awajun fue la que más casos presentó y la que más defunciones por COVID-19 tuvo. Los datos no se encuentran actualizados y no están de libre acceso por lo que su estudio es difícil, sin embargo, los datos que se pueden obtener nos dan un acercamiento a la realidad.

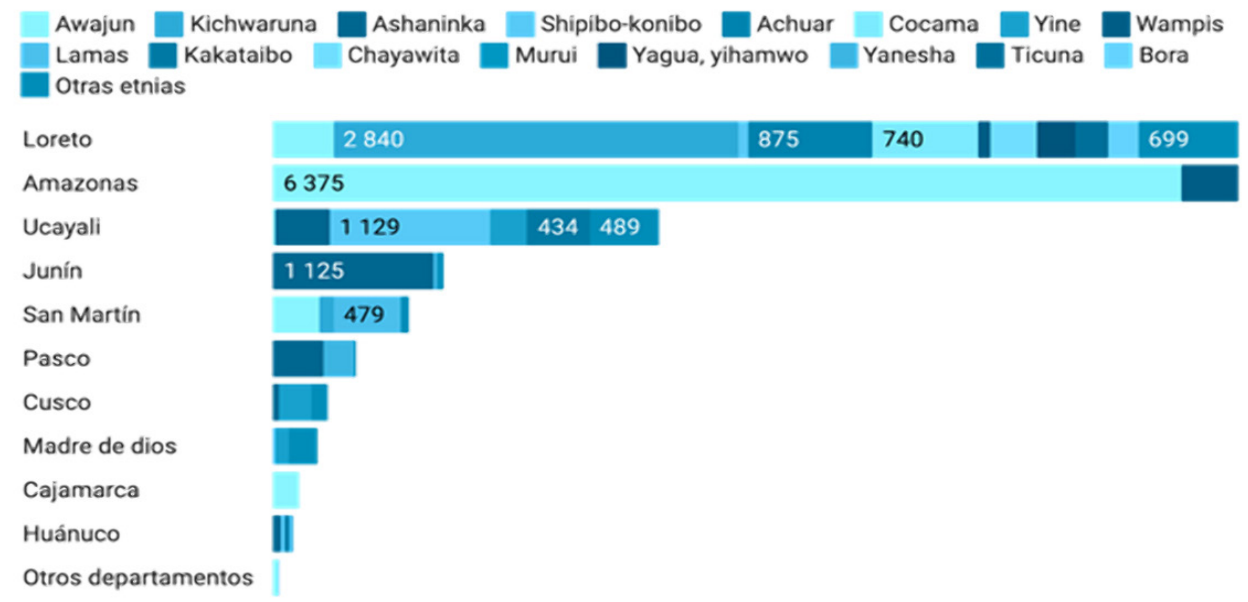

Figura 4. Indígenas de la Amazonía con COVID-19 por regiones (Actualizado a junio 2021).

\section{DISCUSIÓN}

A medida que continúan los bloqueos en numerosos países, sin un cronograma a la vista, los pueblos indígenas que ya enfrentan inseguridad alimentaria, muchos pueblos indígenas que trabajan en ocupaciones tradicionales y economías de subsistencia o en el sector informal se verán afectados negativamente por la pandemia . Perú ya casi iguala en número de casos a Brasil $(56$ 174) en las comunidades amazónicas ${ }^{(13)}$.

Los datos específicos sobre población indígena son muy pobres o no están clasificados en los centros de información de los pacientes que pertenecen a la comunidad pan amazónica. No existen estos sistemas de registros, lo que dificulta el estudio de estas comunidades y la epidemiología de la pandemia en estas regiones geográficas. Los números que muchas veces podemos encontrar son de ONG que muchas veces no son validados. Los gobiernos tienen la intención de contabilizar estos números, pero al verse avasallados por la pandemia, prefieren poner esfuerzos en las grandes urbes.

Es difícil además el estudio de la COVID-19 en las comunidades amazónicas por qué no se registra muchas veces a los pacientes como pertenecientes a estas comunidades, por lo tanto, esta información se pierde, otros muchos no salen de sus comunidades hacia los hospitales o puestos de salud, impidiendo su registro. Los indígenas son tratados en sus mismas comunidades con medicina tradicional, como parte de su cosmovisión.

Los estudios sobre estas comunidades y las comorbilidades que podrían afectar a la COVID-19 son muy pocos por lo que no podemos determinar el estado de salud de estas poblaciones, aunque existen estudios sobre enfermeda- des infecciosas que podrían dar luz sobre el impacto que estas enfermedades podrían causar sobre la población, esto no ocurre con los estudios sobre enfermedades no transmisibles.

Se concluye en que se debe prestar más atención en mejorar el sistema de salud de las comunidades indígenas, esto llevado de la mano de la investigación, y aplicando la medicina en base a la evidencia. Los pueblos indígenas han disminuido su número de casos de la primera ola del año 2020.

Conflictos de interés:Se señala no tener conflictos de interés.

\section{Fuentes de financiamiento: Autofinanciado}

\section{REFERENCIAS BIBLIOGRÁFICAS}

1. Curtice K, Choo E. Indigenous populations: left behind in the COVID-19 response. Lancet. 2020; 395(10239):1753. doi: https://doi.org/10.1016/ S0140-6736(20)31242-3

2. Daniels JP. INPACVIH: tackling HIV in Peru's indigenous communities. Lancet HIV. 2021; 8(5):e255. doi: 10.1016/S2352-3018(21)00077-1.

3. Amigo I. Indigenous communities in Brazil fear pandemic's impact. Science. 2020;368(6489):352. doi: 10.1126/science.368.6489.352.

4. Iglesias-Osores S, Saavedra-Camacho JL. COVID-19 en comunidades indígenas del Perú: casos y accesibilidad a servicios de salud. An Fac Med. 2020;81(2):250-1. doi: 10.15381/anales.v81i2.18057

5. Saxton AT, Stanifer JW, Miranda JJ, Ortiz EJ, Taype-Rondan A, Pan W. Prevalence and Associated Risk Factors of Diabetes, Chronic Kidney Disease, and Hypertension of Adults in the Peruvian Amazon: The Amarakaeri Reserve Cohort Study. SSRN Electron J. 2020. doi: 10.2139/ ssrn.3546039

6. Russell NK, Nazar K, Del Pino S, Gonzalez MA, Díaz Bermúdez XP, Ravasi G. HIV, syphilis, and viral hepatitis among Latin American indigenous peoples and Afro-descendants: A systematic review. Rev Panam 
Salud Publica.2020;43:e17. doi: 10.26633/RPSP.2019.17.

7. Carr A. COVID-19, indigenous peoples and tourism: a view from New Zealand. Tour Geogr.2020;22(3):491-502. doi: 10.1080/14616688.2020.1768433

8. Montag D, Barboza M, Cauper L, Brehaut I, Alva I, Bennett A, et al. Healthcare of Indigenous Amazonian Peoples in response to COVID-19: Marginality, discrimination and revaluation of ancestral knowledge in Ucayali, Peru. [Internet]. Vol. 6, BMJ Glob Health. 2021;6(1):4479. doi: 10.1136/ bmjgh-2020-004479.

9. Adalja AA, Toner E, Inglesby T V. Priorities for the US Health Community Responding to COVID-19. JAMA. 2020;323(14):1343-4. doi: 10.1001/ jama.2020.3413

10. Meneses-Navarro S, Freyermuth-Enciso MG, Pelcastre-Villafuerte BE, Campos-Navarro R, Meléndez-Navarro DM, Gómez-Flores-Ramos L. The challenges facing indigenous communities in Latin America as they confront the COVID-19 pandemic. Int J Equity Health. 2020;19(1):63. doi: 10.1186/ s12939-020-01178-4

11. Zavaleta C. COVID-19: review Indigenous peoples' data. Nature. 2020; 580(7802):185. doi: 10.1038/d41586-020-01032-1.

12. UNDESA. COVID-19 and Indigenous peoples | United Nations For Indigenous Peoples [Internet]. 2020 [citado 27 de junio de 2021]. Disponible en: https://www.un.org/development/desa/indigenouspeoples/covid-19.html

13. APIB. COVID-19 e os Povos Indígenas [Internet]. Articulação dos Povos Indígenas do Brasil (Apib). 2020 [citado 27 de junio de 2021]. Disponible en: https://covid19.socioambiental.org/ 